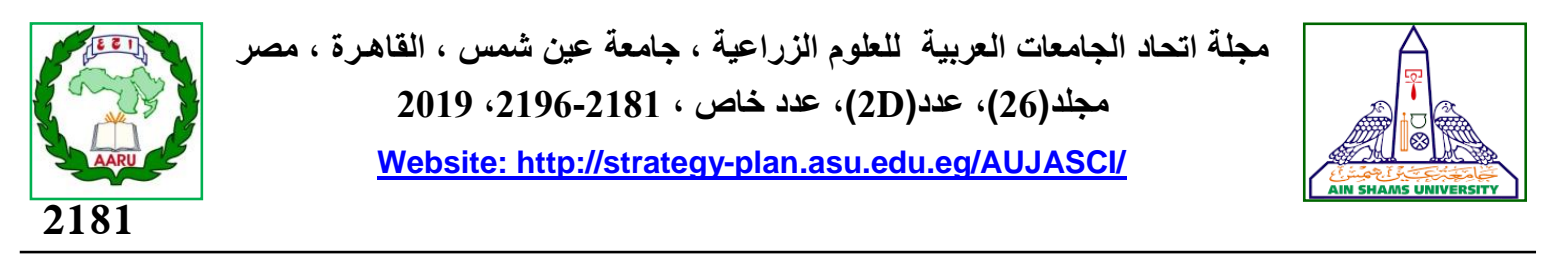

نموذج اقتصادي قياسي لمحدات الموارد المائية المصرية

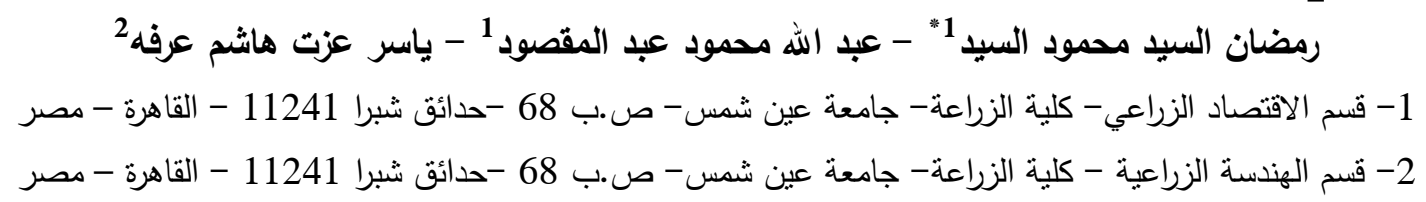

${ }^{*}$ Corresponding author: ramdan.alsyd2000@gmail.com

Received 26 June, 2018

Accepted 28 August, 2018

المحاصيل الاستراتيجية ذات معدلات استهلاك مائي

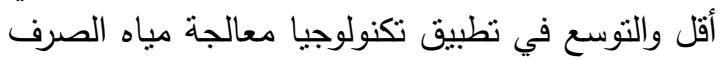

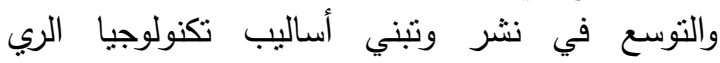

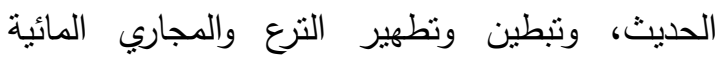
وغيرها من السياسات المائية الأخرى ولاسيما في الآنيانة

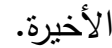

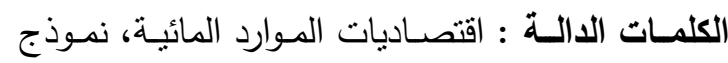
اقتصادي قياسي آني، السياسات المائية.

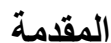

تعتبر الموارد المائية هي المورد الطبيعي الأساسي

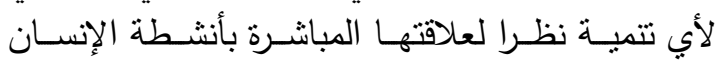

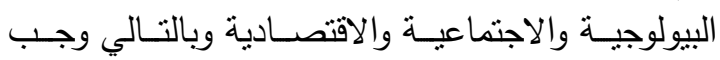

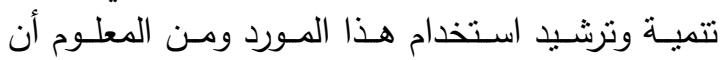

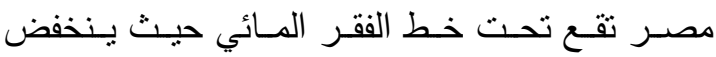

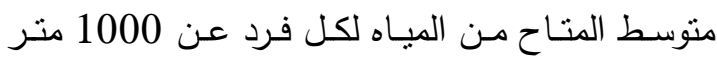

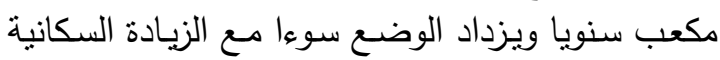

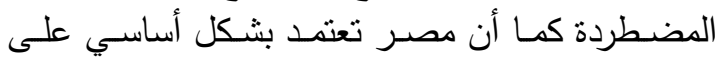

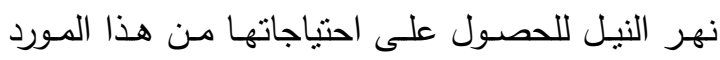

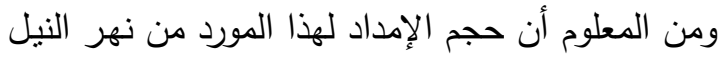

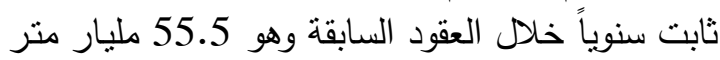

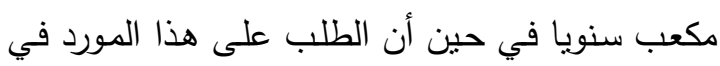

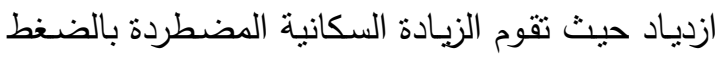

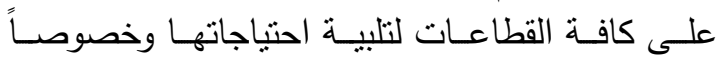




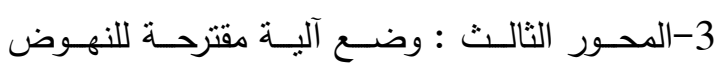

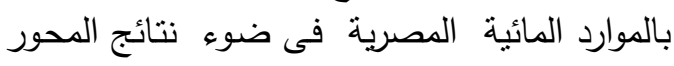

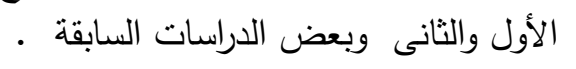

\section{الطريقة البحثية ومصادر البيانات}

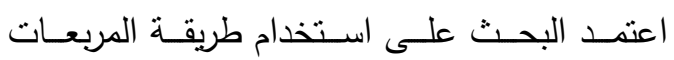

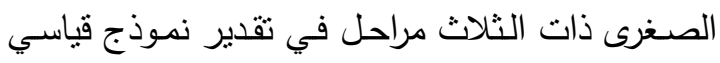

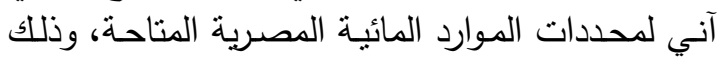
إعنماداً على برامج التحليل الاحصائي منل ( EViews 9. (SPSS18

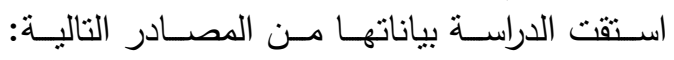
البيانات الثانوية الصادرة من الجهات الرسمية كالنشرات النات التهات

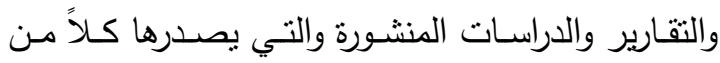

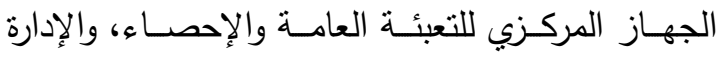

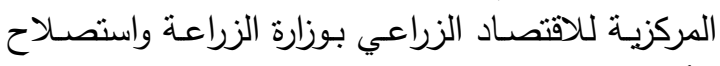

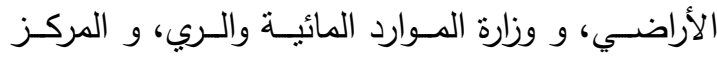

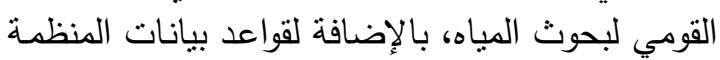

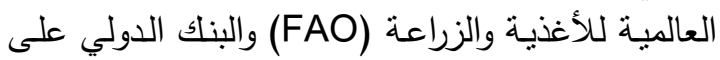
الشبكة الدولية للمعلومات ل لاغلة ولزاعة العات

\section{مناقشة نتائج البحث}

1- المحور الأول: حصر أهم محددات الموارد المائية في مصر باستخدام النماذج الآنية

نتائج التقدير الإحصائي للنموذج الاقتصادي القياسي الآني لمحددات الموارد المائية المصرية في ظل المائهادي أثنير

السياسات المائية

نماذج المعادلات الانية هي النماذج التي لا يمكن

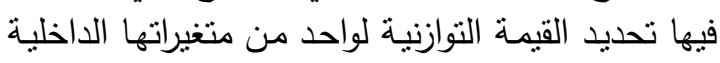

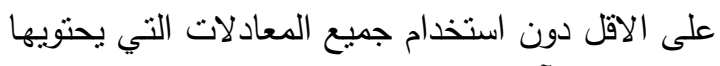

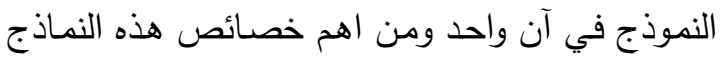

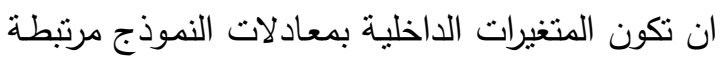

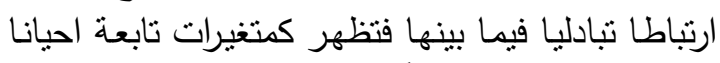

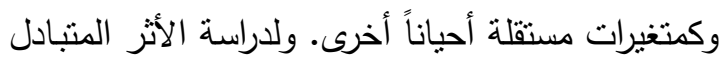

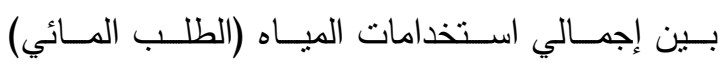

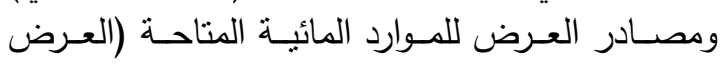

القطـاع الزراعـي الــي ينطلـب منــة تلبيـة احتياجـات

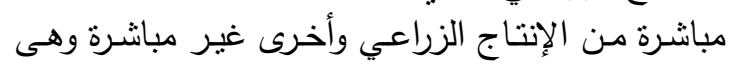

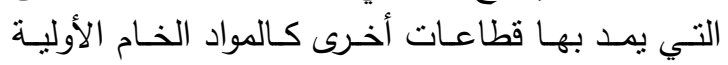

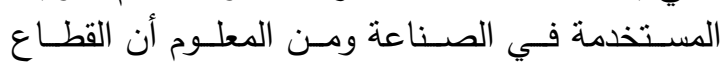

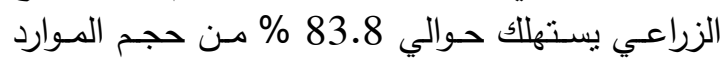

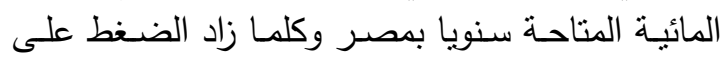

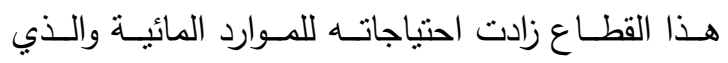

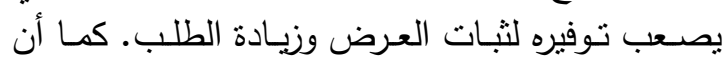

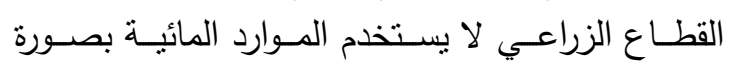

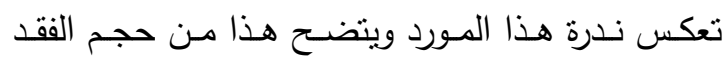

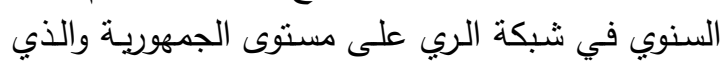

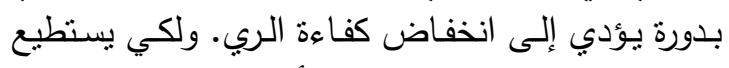

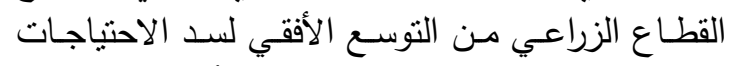

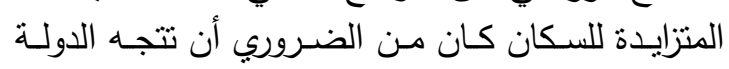

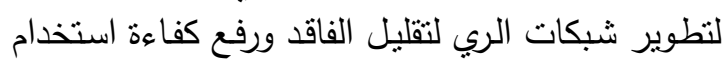

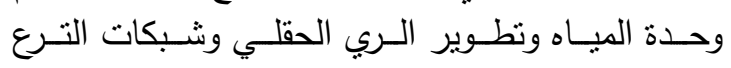

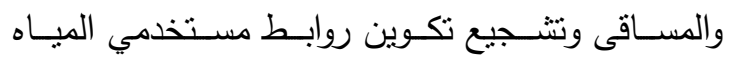

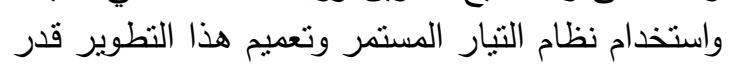

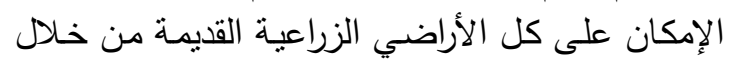

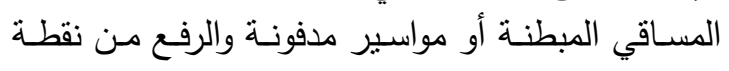

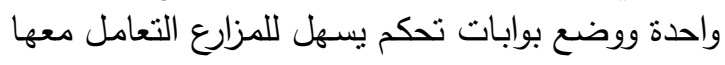

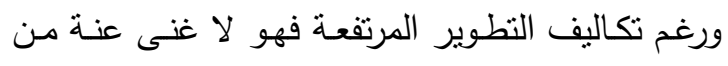

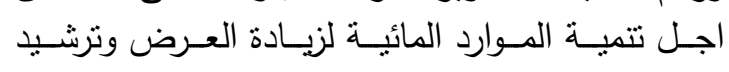

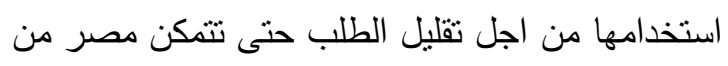
تحقيق التتمية المطلوبة.

\section{مشكلة وهدف البحث}

نظراً لان قضية المياه اصبحت قضية الساعة والتي استحوذت على كافة الجهود المصرية لمحاولـة تذليل

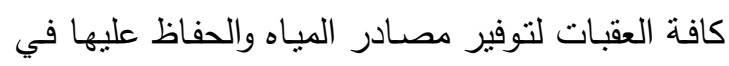

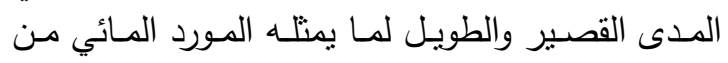

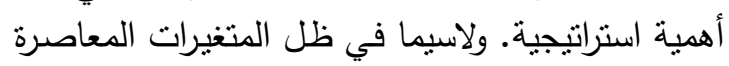

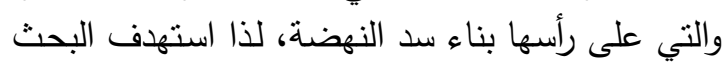
تحقيق المحاور التالية : 1- المحسيق المحاور الأول : يتمثنل في حصـر أهم محددات

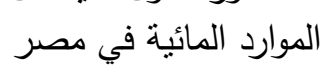

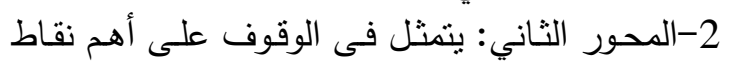

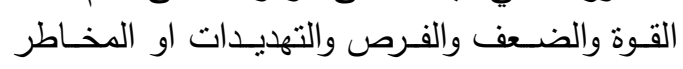

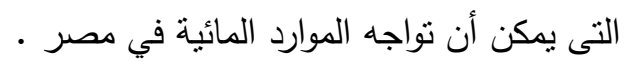




$$
\begin{aligned}
& \text { • معادلة الطلب المائي المصري (معادلة سلوكية) } \\
& Y_{2}=\alpha_{10}+\alpha_{7} X_{7}+\alpha_{9} X_{9}+\alpha_{13} X_{13}+\alpha_{14} X_{14}+ \\
& \alpha_{22} X_{22}+\alpha_{24} X_{24}+\alpha_{25} X_{25}+\alpha_{26} X_{26}+\alpha_{11} Y_{1} \\
& \text { • معادلة التوازن المائي ( معادلة تعريفية ) } \\
& Y_{1}=Y_{2}
\end{aligned}
$$$$
\text { حيث : }
$$

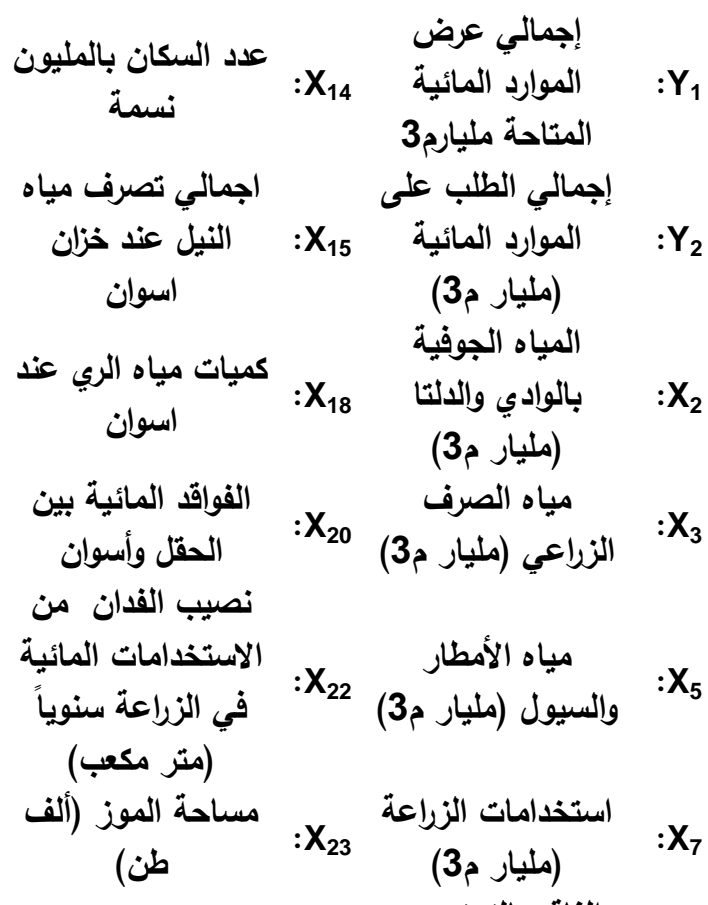

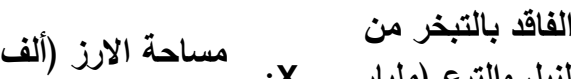
طن) النيل والترع (مليار من X م)

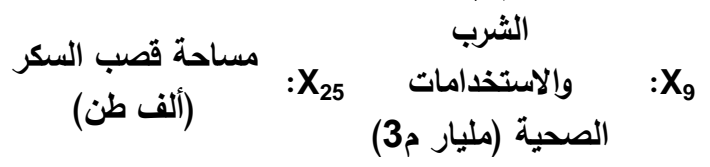

مساحة المحاصيل

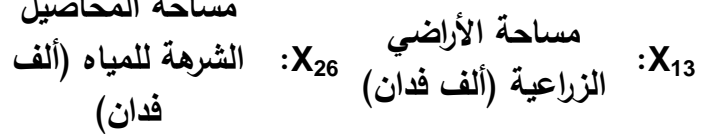

متغير انتقالي يشير إلى تأثير السياسات المائية

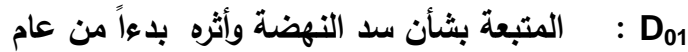

\section{4}

المائي) وتم تقدير النموذج الاقتصـادي القياسي الآني

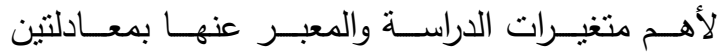

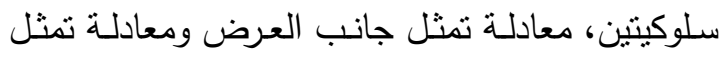

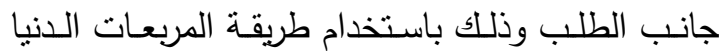

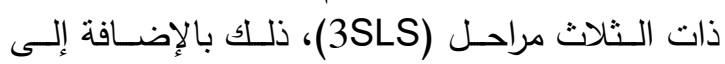

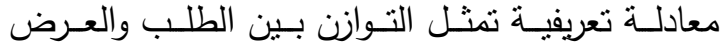
المائي.

توصيف النموذج الاقتصادي القياسي الأني لمحددات

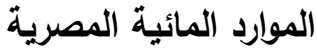

أولاً: في حالة عدم وجود تأثثر السياسات المائية • معادلة العرض المائي المصري ( معادلة سلوكية) $Y_{1}=\alpha_{0}+\alpha_{2} X_{2}+\alpha_{3} X_{3}+\alpha_{5} X_{5}+\alpha_{15} X_{15}+$ $\alpha_{18} X_{18}+\alpha_{20} X_{20}$

• معادلة الطلب المائي المصري (معادلة سلوكية) $Y_{2}=\alpha_{10}+\alpha_{7} X_{7}+\alpha_{9} X_{9}+\alpha_{13} X_{13}+\alpha_{14} X_{14}+$ $\alpha_{22} X_{22}+\alpha_{24} X_{24}+\alpha_{25} X_{25}+\alpha_{26} X_{26}+\alpha_{11} Y_{1}$

• معادلة النوازن المائي ( معادلة تعريفية ) $\mathrm{Y}_{1}=\mathrm{Y}_{2}$

ثانياً: في حالة وجود تأثير السياسات المائية (متغير (نتقالي) • معادلة العرض المائي المصري ( معادلة سلوكية) $Y_{1}=\alpha_{0}+\alpha_{2} X_{2}+\alpha_{3} X_{3}+\alpha_{5} X_{5}+\alpha_{15} X_{15}+$ $\alpha_{18} X_{18}+\alpha_{20} X_{20}+\alpha_{01} D_{01}$ 
زيادة في كلا من اجمالي تصرف مياه النيل عند

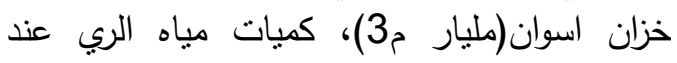

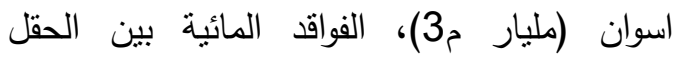

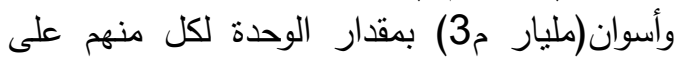

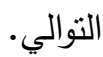

• كما اثارت النتائج إلى تزايد الطلب على الموارد المائية المتاحة بنحو 0.425 1.425، 0.032، 0.949، 0.017،

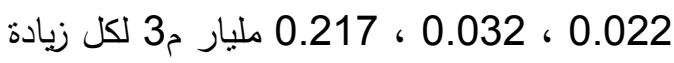

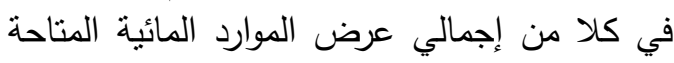

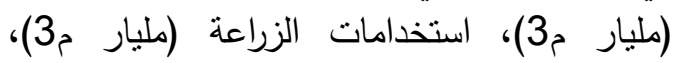

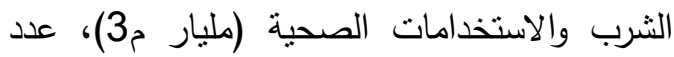

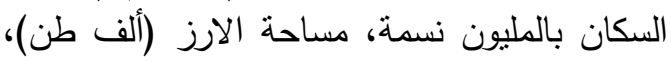

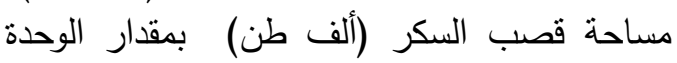
لكل منهم على التوالي. • في حين يتتاقص الطلب لئل على الموارد المائية المتاحة بنحو 0.004، 0.005، م3 لكل زيادة في كلاً من مساحة الأراضي الزراعية

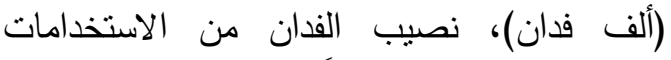

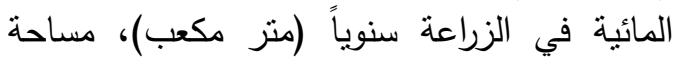
المحاصيل الثرهة للمياه (ألف فدان) بمقدار الوحدة لكنة لكل منهم على التوالي. وقد ثنت المعنوية الاحصائية عند مسنوى يتراوح

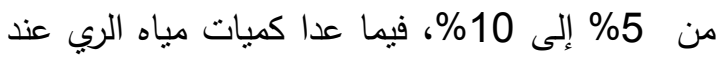

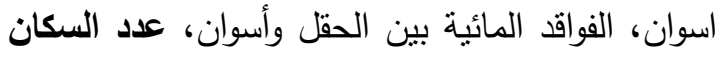

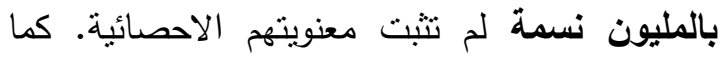

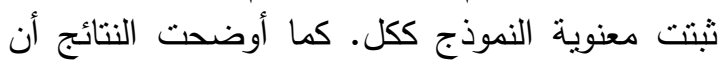

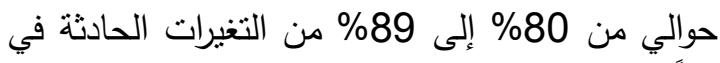
كلاً من إجمالي العرض من المب الموارد المائية وإجمالي الئي

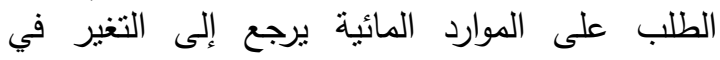
المتغيرات المستقلة محل الدراسة.
أولاً: نتائج النموذج القياسي الأني لمحددات الموارد المائية المصرية نتانج * * حالة عدم وجود تأثير السياسات المائية • معادلة العرض المائي المصري ( معادلة سلوكية): $Y_{1}=56.56+1.122 X_{2}+1.018 X_{3}+1.296 X_{5}-$ $0.003 X_{15}-0.017 X_{18}-0.007 X_{20}$

$\mathrm{R}^{2}$ Adjusted $=0.888$

D.W $=1.83$

• معادلة الطلب المائي المصري (معادلة سلوكية): $Y_{2}=35.01+1.425 X_{7}+0.949 X_{9}-0.004 X_{13}$ $+0.017 X_{14}-0.006 X_{22}+0.023 X_{24}+0.032 X_{25}$ $-0.022 X_{26}+0.217 Y_{1}$

$\begin{array}{ll}\mathrm{R}_{\text {Adjusted }}=0.798 & \mathrm{D} . \mathrm{W}=2.17\end{array}$ اوضحت نتائج التقدير الاحصائي للنموذج القياسي الآني لمحددات الموارد المائية المصرية خلائل الفئ الفترة

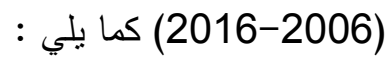

• اشتارت النتائج إلى تزايد المعروض من الموارد المائية المتاحة بنحو 1.122 ، 1.1818 ل 1.018 ، 1.29 مليار م3 لكل زيادة في كلا من المياه الجوفية

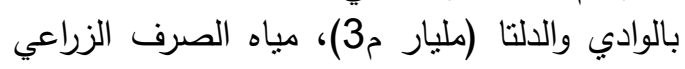

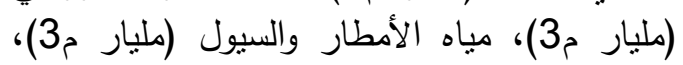

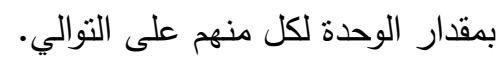

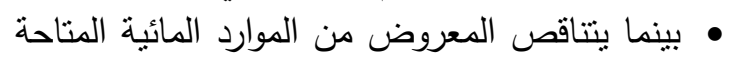

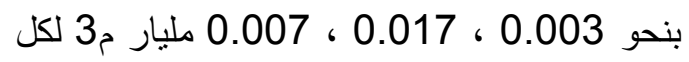




$$
\text { جدول 1. نتائج النموذج القياسي الأني لمحددات الموارد المائية الدصرية }
$$

System: UNTITLED

Estimation Method: Three-Stage Least Squares

Date: 05/24/18 Time: 16:12

Sample: 111

Included observations: 11

Total system (balanced) observations 22

Linear estimation after one-step weighting matrix

\begin{tabular}{|c|c|c|c|c|}
\hline & Coefficient & Std. Error & t-Statistic & Prob. \\
\hline $\mathrm{C}(1)$ & 56.55689 & 1.843887 & 30.67265 & 0.0000 \\
$\mathrm{C}(3)$ & 1.121575 & 0.107281 & 10.4554 & 0.0001 \\
$\mathrm{C}(4)$ & 1.018397 & 0.029717 & 34.27021 & 0.0000 \\
$\mathrm{C}(6)$ & 1.295649 & 0.242239 & 5.348643 & 0.0031 \\
$\mathrm{C}(7)$ & -0.003073 & 0.013629 & -0.225462 & 0.0305 \\
$\mathrm{C}(8)$ & -0.017448 & 0.008643 & -2.018765 & 0.0995 \\
$\mathrm{C}(9)$ & -0.007205 & 0.026167 & -0.275346 & 0.7941 \\
$\mathrm{C}(10)$ & 35.01032 & 67.12943 & 0.521535 & 0.6243 \\
$\mathrm{C}(11)$ & 1.424546 & 0.994787 & 1.432011 & 0.2116 \\
$\mathrm{C}(12)$ & 0.949921 & 0.050395 & 18.84939 & 0.0000 \\
$\mathrm{C}(13)$ & -0.004231 & 0.007277 & -0.581431 & 0.0562 \\
$\mathrm{C}(14)$ & 0.016877 & 0.014982 & 1.126484 & 0.3111 \\
$\mathrm{C}(15)$ & -0.005656 & 0.009204 & -0.614503 & 0.0458 \\
$\mathrm{C}(16)$ & 0.022657 & 0.010346 & 2.189996 & 0.0801 \\
$\mathrm{C}(17)$ & 0.032318 & 0.012972 & 2.491300 & 0.0551 \\
$\mathrm{C}(18)$ & -0.022386 & 0.010286 & -2.176407 & 0.0815 \\
$\mathrm{C}(19)$ & 0.217008 & 0.060922 & 3.562040 & 0.0162 \\
\hline
\end{tabular}

Equation: $\mathrm{Y} 1=\mathrm{C}(1)+\mathrm{C}(3)^{*} \mathrm{X} 2+\mathrm{C}(4)^{*} \mathrm{X} 3+\mathrm{C}(6)^{*} \mathrm{X} 5+\mathrm{C}(7)^{*} \mathrm{X} 15+\mathrm{C}(8)^{*} \mathrm{X} 18+\mathrm{C}(9){ }^{*} \mathrm{X} 20$

Instruments: $\mathrm{X} 2 \times 3 \times 4 \times 5 \times 7 \times 8 \times 9 \times 13 \times 14 \times 15 \times 20$

Observations: 11

\begin{tabular}{|l|l|l|l|}
\hline R-squared & 0.899429 & Mean dependent var & 73.62637 \\
Adjusted R-squared & 0.888572 & S.D. dependent var & 2.382550 \\
S.E. of regression & 0.090046 & Sum squared resid & 0.032433 \\
Durbin-Watson stat & 1.831583 & & \\
\hline
\end{tabular}

Equation: $\mathrm{Y} 2=\mathrm{C}(10)+\mathrm{C}(11)^{*} \mathrm{X} 7+\mathrm{C}(12)^{*} \mathrm{X} 9+\mathrm{C}(13)^{*} \mathrm{X} 13+\mathrm{C}(14)^{*} \mathrm{X} 14+\mathrm{C}(15)^{*} \mathrm{X} 22$ $+C(16)^{*} X 24+C(17)^{*} X 25+C(18)^{*} X 26+C(19)^{*} Y 1$

Instruments: X2 X3 X4 X5 X7 X8 X9 X13 X14 X15 X20

Observations: 11

\begin{tabular}{|l|l|c|c|}
\hline R-squared & 0.799877 & Mean dependent var & 73.66909 \\
Adjusted R-squared & 0.798775 & S.D. dependent var & 3.023033 \\
S.E. of regression & 0.105816 & Sum squared resid & 0.011197 \\
Durbin-Watson stat & 2.170165 & & \\
\hline
\end{tabular}

المصدر: حسبت من خلال البرنامج الإحصائي (EViews 9) 
الآونة الأخيرة. ولعل من أهم هذه السياسات: تقليص

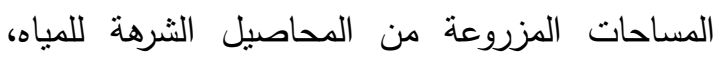

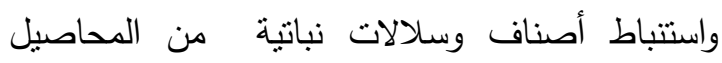
الاستراتيجية ذات معدلات استهلاك مائي أقل والتوسع التهات

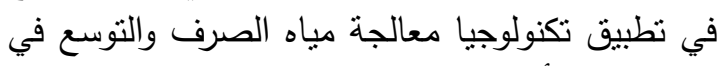

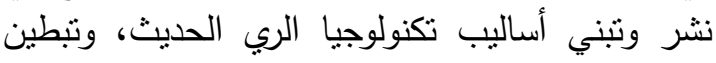

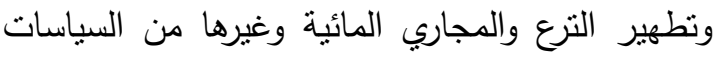
المائية الأخرى ولاسيما في الآونة الأخيرة.

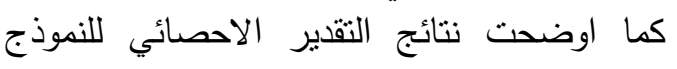

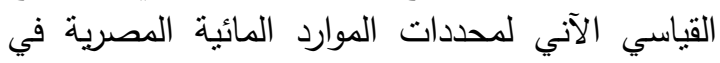
ظل تأثثير السياسات المائية في الآونة الأخيرة خلال الفترة (2006-2016) ما يلي : بأير اليبانيات

• انشارت النتائج إلى تزايد المعروض من الموارد 1.334 ، 1.009 ،

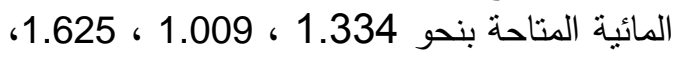
0.013 ، 0.018 مليار م3 لكل زيادة في كلا من المياه الجوفية بالوادي والدلتا (مليار م3 مليار)، مياه

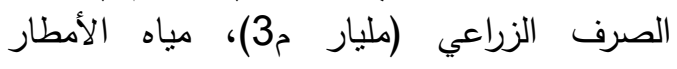
والسيول (مليار م3)، اجمالي تصرف مياه النيل

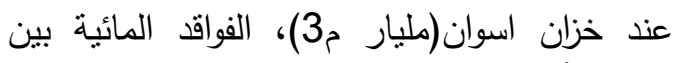

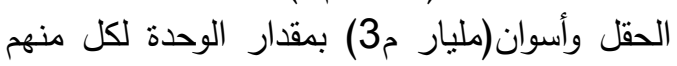
على التوالي. • بينما يتناقص المعروض من المبالم الموارد المائية المتاحة

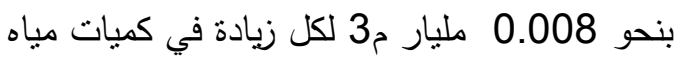

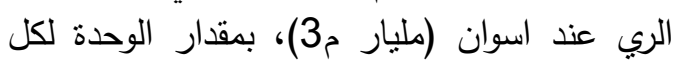

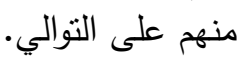

ثانياً: نتائج النموذج القياسي الآني لمحددات الموارد المائية المصرية في ظل تأثير السياني الأنيات الميات المائية في الآونة الأخيرة

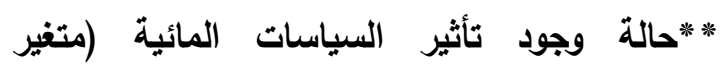
(نتقالي)

• معادلة العرض المائي المصري ( معادلة سلوكية): $Y_{1}=53.28+1.334 X_{2}+1.009 X_{3}+1.625 X_{5}$ $+0.013 X_{15}-0.009 X_{18}+0.018 X_{20}$ $+0.213 D_{01}$

$\mathrm{R}_{\text {Adjusted }}^{2}=0.689 \quad$ D. $W=2.68$

• معادلة الطلب المائي المصري (معادلة سلوكية) $Y_{2}=38.83+1.515 X_{7}+0.943 X_{9}-0.005 X_{13}$ $+0.019 X_{14}-0.006 X_{22}+0.021 X_{24}+0.031 X_{25}$ $-0.021 X_{26}+0.211 Y_{1}$

$\mathrm{R}^{2}{ }_{\text {Adjusted }}=0.788 \quad \mathrm{D} . \mathrm{W}=2.16$

تشير نتائج التقدير الإحصائي إلى أن المعروض المباد

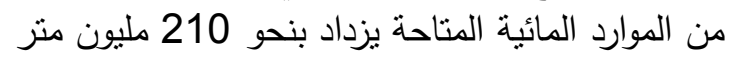

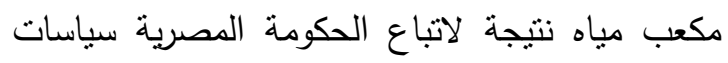

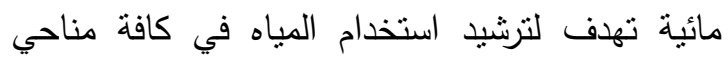

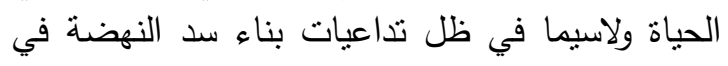


الأخيرة 2. نتائج النموذج القياسي الأني لمحددات الموارد المائية المصرية في ظل تأثثر السياسات المائية في الأونة

System: UNTITLED

Estimation Method: Three-Stage Least Squares

Date: 05/24/18 Time: 17:09

Sample: 111

Included observations: 11

Total system (balanced) observations 22

Linear estimation after one-step weighting matrix

\begin{tabular}{|c|c|c|c|c|}
\hline & Coefficient & Std. Error & t-Statistic & Prob. \\
\hline $\mathrm{C}(1)$ & 53.28051 & 1.231169 & 43.27636 & 0.0000 \\
$\mathrm{C}(3)$ & 1.334979 & 0.075650 & 17.64675 & 0.0001 \\
$\mathrm{C}(4)$ & 1.008673 & 0.017174 & 58.73096 & 0.0000 \\
$\mathrm{C}(6)$ & 1.624685 & 0.153777 & 10.56519 & 0.0005 \\
$\mathrm{C}(7)$ & 0.013302 & 0.008425 & 1.578833 & 0.1895 \\
$\mathrm{C}(8)$ & -0.008878 & 0.005129 & -1.730907 & 0.1585 \\
$\mathrm{C}(9)$ & 0.018217 & 0.015515 & 1.174126 & 0.3055 \\
$\mathrm{C}(20)$ & 0.213505 & 0.043661 & 4.890039 & 0.0081 \\
$\mathrm{C}(10)$ & 38.83321 & 67.01210 & 0.579496 & 0.5933 \\
$\mathrm{C}(11)$ & 1.514856 & 0.992831 & 1.525794 & 0.2018 \\
$\mathrm{C}(12)$ & 0.942746 & 0.050196 & 18.78120 & 0.0000 \\
$\mathrm{C}(13)$ & -0.004799 & 0.007263 & -0.660709 & 0.5449 \\
$\mathrm{C}(14)$ & 0.019221 & 0.014868 & 1.292764 & 0.2657 \\
$\mathrm{C}(15)$ & -0.006245 & 0.009188 & -0.679649 & 0.5340 \\
$\mathrm{C}(16)$ & 0.021425 & 0.010329 & 2.074216 & 0.1067 \\
$\mathrm{C}(17)$ & 0.031127 & 0.012955 & 2.402693 & 0.0741 \\
$\mathrm{C}(18)$ & -0.021162 & 0.010269 & -2.060703 & 0.1084 \\
$\mathrm{C}(19)$ & 0.210867 & 0.060849 & 3.465406 & 0.0257 \\
\hline
\end{tabular}

Equation: $Y 1=C(1)+C(3)^{\star} X 2+C(4)^{\star} X 3+C(6)^{*} X 5+C(7)^{*} X 15+C(8)^{*} X 18+C(9)$ ${ }^{*} \mathrm{X} 20+\mathrm{C}(20){ }^{*} \mathrm{D} 01$

Instruments: X2 X3 X4 X5 X7 X8 X9 X13 X14 X15 X20

Observations: 11

\begin{tabular}{|l|l|c|l|}
\hline R-squared & 0.699809 & Mean dependent var & 73.62637 \\
Adjusted R-squared & 0.689364 & S.D. dependent var & 2.382550 \\
S.E. of regression & 0.060065 & Sum squared resid & 0.010823 \\
Durbin-Watson stat & 2.683771 & & \\
\hline
\end{tabular}

Equation: $Y 2=C(10)+C(11)^{*} X 7+C(12)^{*} X 9+C(13)^{*} X 13+C(14)^{*} X 14+C(15)^{*} X 22$ $+\mathrm{C}(16)^{*} \mathrm{X} 24+\mathrm{C}(17)^{*} \mathrm{X} 25+\mathrm{C}(18)^{*} \mathrm{X} 26+\mathrm{C}(19)^{*} \mathrm{Y} 1$

Instruments: X2 X3 X4 X5 X7 X8 X9 X13 X14 X15 X20

Observations: 11

\begin{tabular}{|l|l|l|l|}
\hline R-squared & 0.799874 & Mean dependent var & 73.66909 \\
Adjusted R-squared & 0.788741 & S.D. dependent var & 3.023033 \\
S.E. of regression & 0.107273 & Sum squared resid & 0.011507 \\
Durbin-Watson stat & 2.164727 & & \\
\hline
\end{tabular}

المصدر: حسبت من خلال البرنامج الإحصائي (EViews 9) 
اسوان، الفواقد المائية بين الحقل وأسوان، عدد السكان

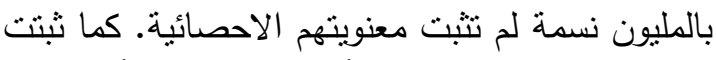

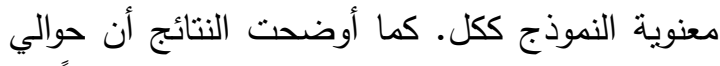

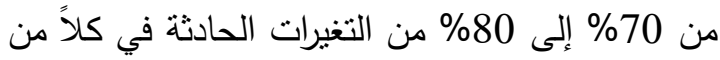

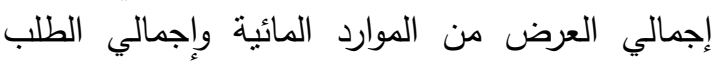
على الموارد المائية يرجع إلى التغير في المتئي المتيرات الميرات المستقلة محل الدراسة.

2- المحور الثاني: الوقوف على أهم نقاط القوة

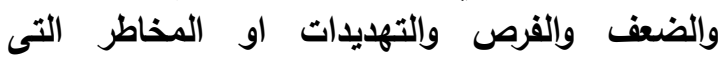
يمكن أن تواجه الموارد المائية في مصر باستخدام SWOT Analysis

أهم نقاط القوة والضعف والفرص والتهديدات التي تواجه قطاع الموارد المائية المصرية أولاً : نقاط القوة ونقاط الضعف التي تواجه الموارد المائية المصرية : لماطة
• كما اثنارت النتائج إلى تزايد الطلب على الموارد المائية المتاحة بنحو 0.021 0.514، 0.942، 0.919، 0.019،

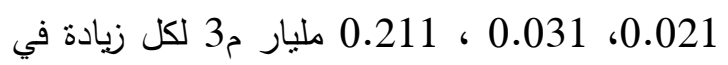
كلا من إجمالي عرض الموارد المائية المتاحة (مليار

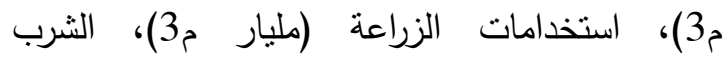

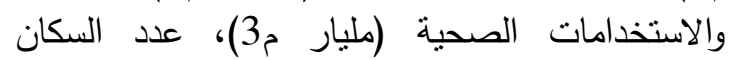

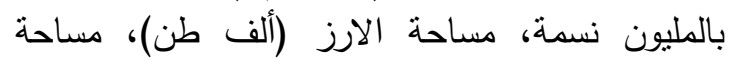

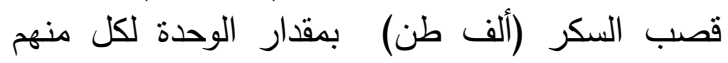
على التوالي. • في حين ينتاقص الطلب على الموارد المائية

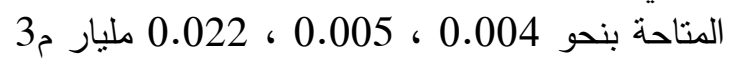
لكل زيادة في كلاً من مساحة الأراضي الزراعية (ألف

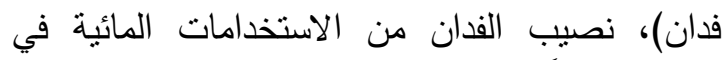
الزراعة سنوياً (متر مكعب)، مساحة المحاصيل الثرهة

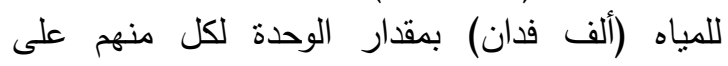

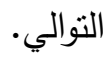
وقد ثنتت المعنوية الاحصائية عند مستوى يتراوح من 5\% إلى 10\%، فيما عدا كميات مياه الري عند

\begin{tabular}{|c|c|}
\hline نقاط الضعف & نقاط القوة \\
\hline 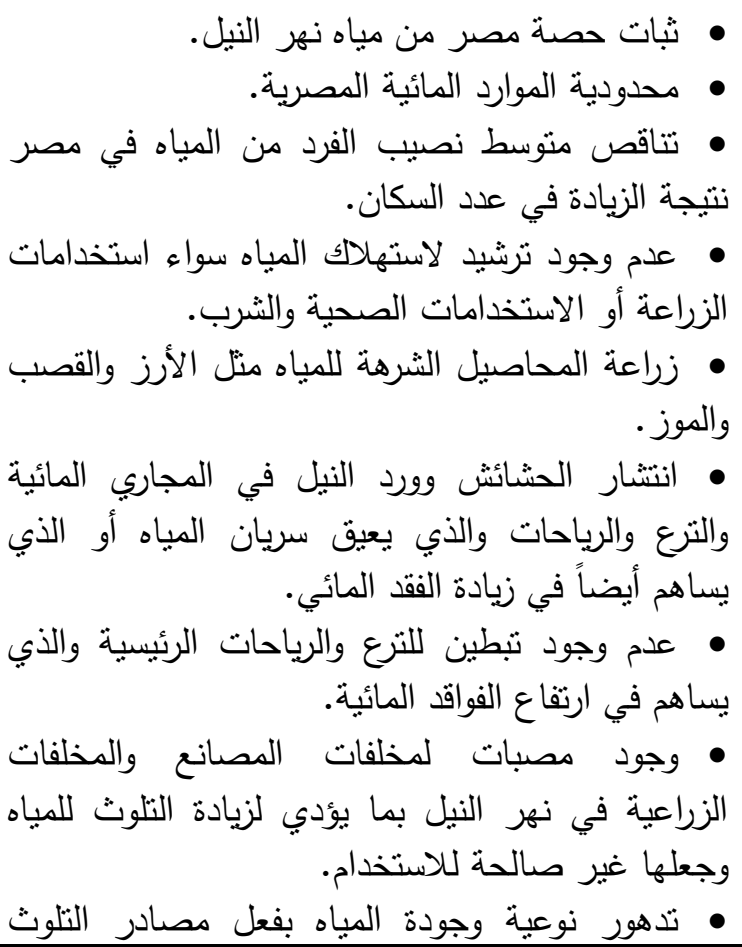 & 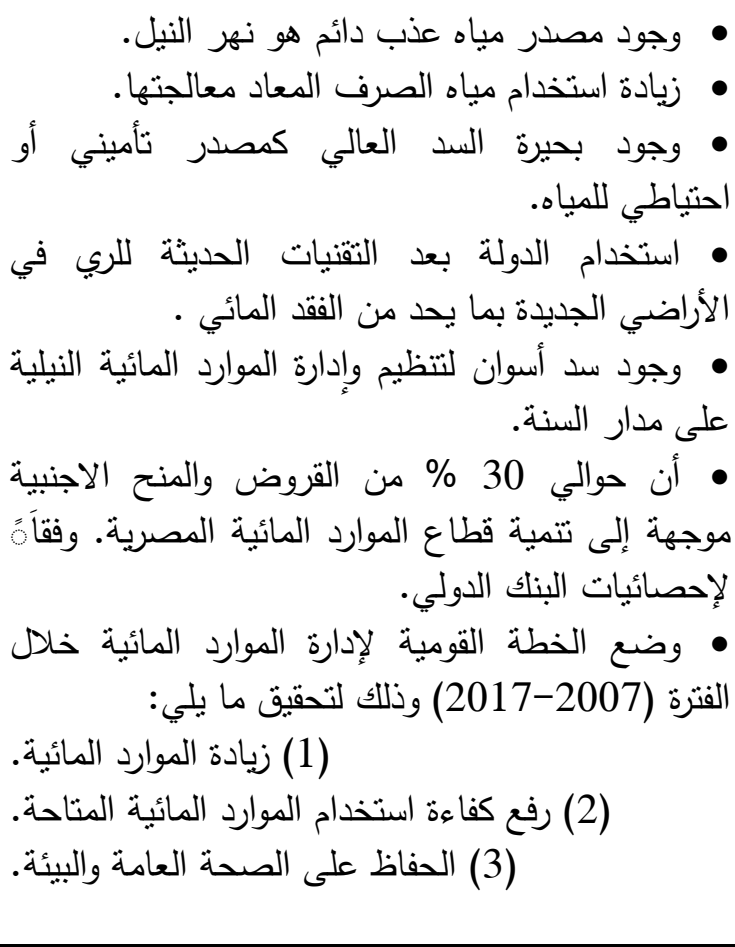 \\
\hline
\end{tabular}




\begin{tabular}{|c|c|}
\hline نقاط الضعف & نقاط القوة \\
\hline 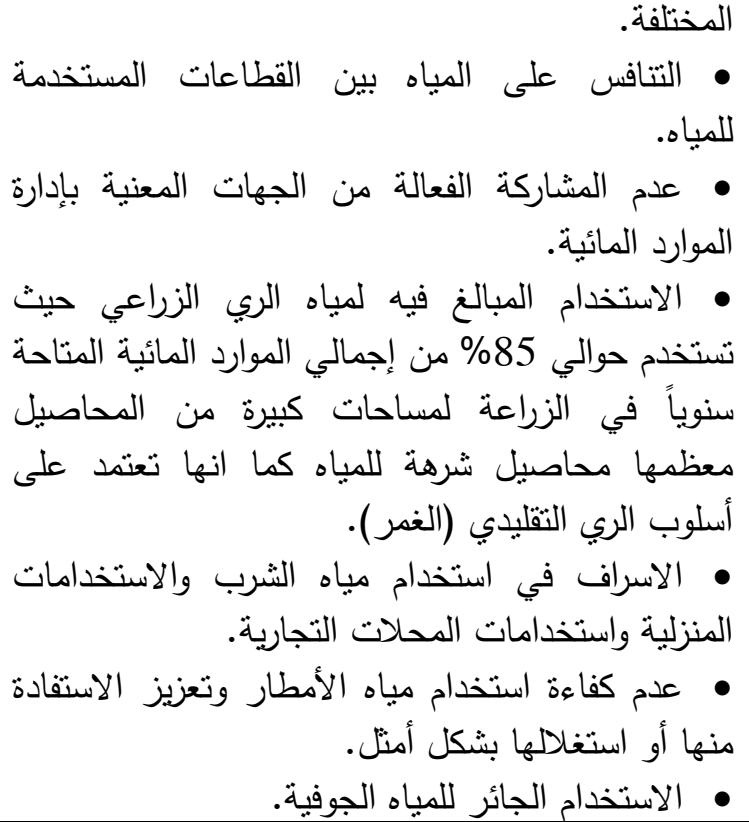 & \\
\hline
\end{tabular}

المصدر: الدراسات المرجعية السابقة بالإضافة إلى المقابلات الثخصية مع بعض الجهات المعنية.

ثانياً: الفرص والتهديدات (المخاطر) التي تواجه الموارد المائية المصرية

\begin{tabular}{|c|c|}
\hline التهديدات (المخاطر) & الفرص \\
\hline 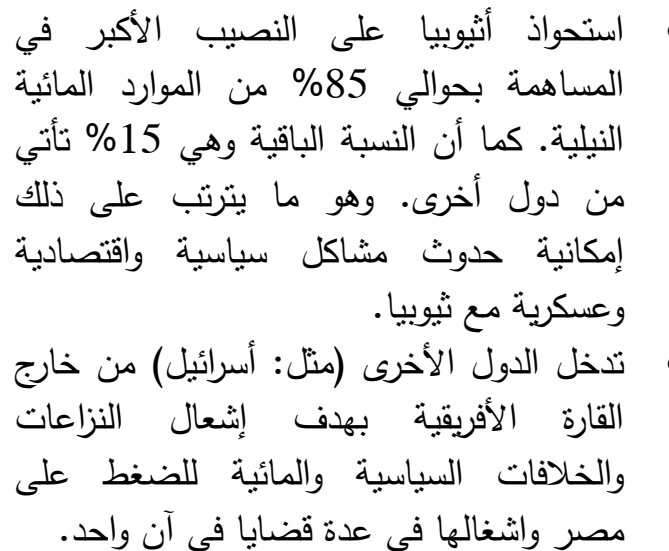 & 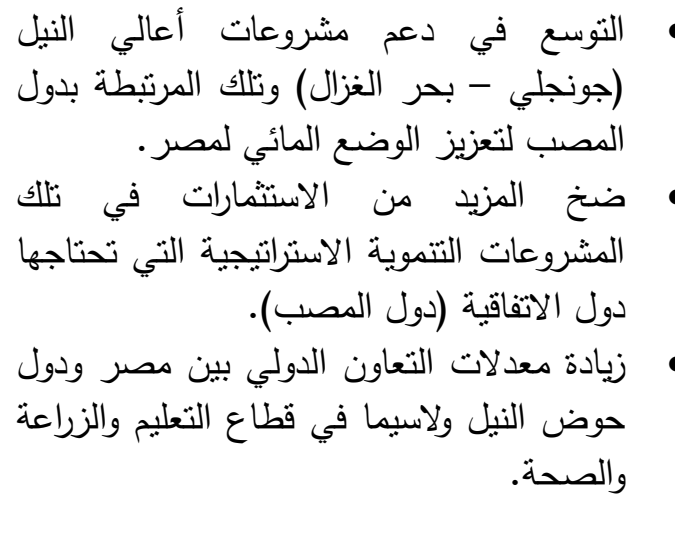 \\
\hline
\end{tabular}

المصدر: الدراسات المرجعية السابقة بالإضافة إلى المقابلات الثحصية مع بعض ممنلى الجهات المعنية. 
3- المحور الثالث: وضع آلية مقترحة للنهوض فئل

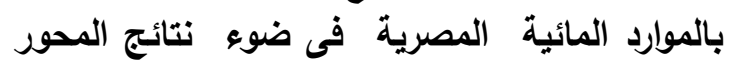
الأول والثانى ويعض الدانة الدراسات السابقة.

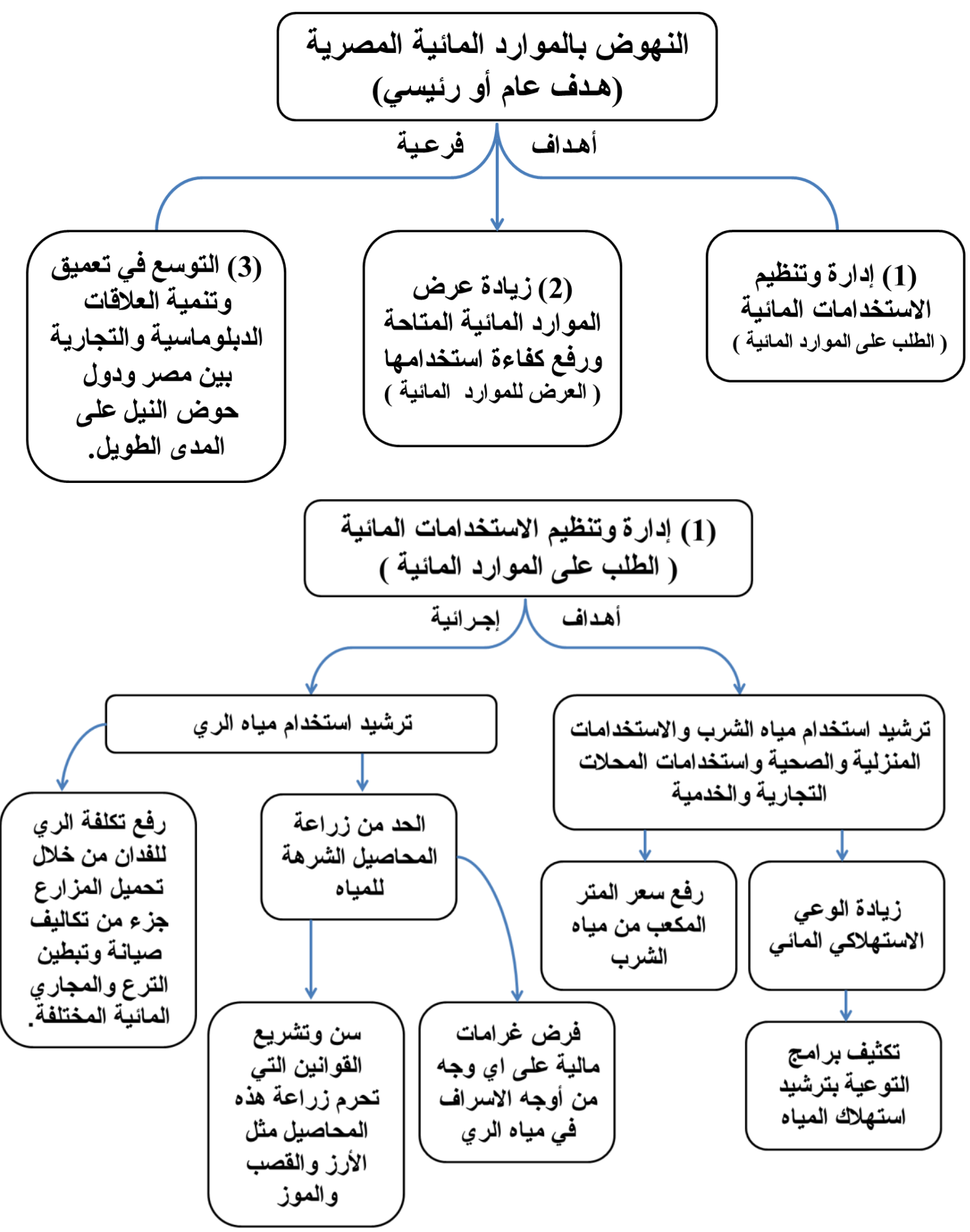




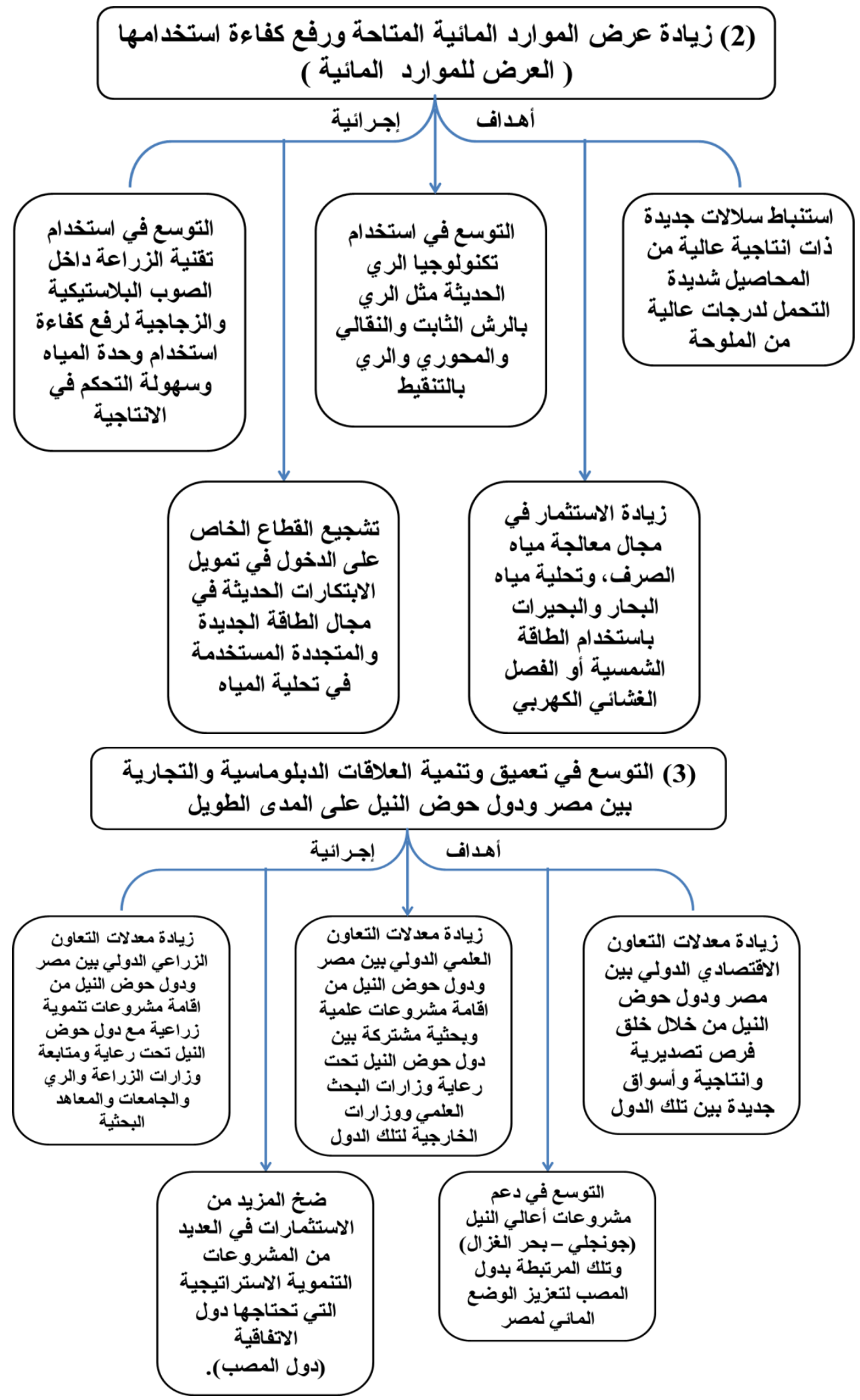

شكل 1. آلية مقترحة للنهوض بالموارد المائية المصرية 


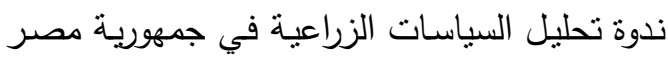

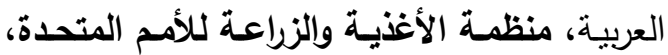

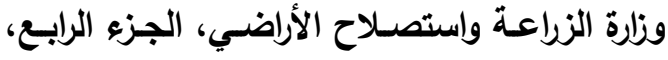

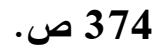
مرسسى، سـعيد عبـدالفتاح عنـاني 2017. " الآثار

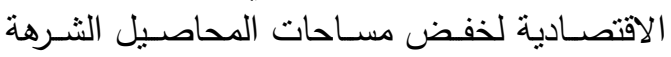

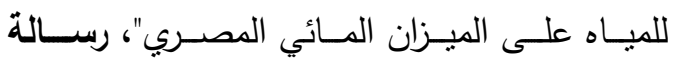

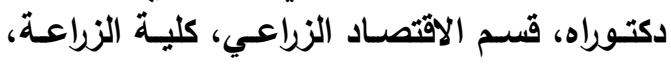

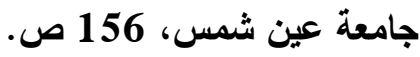

$$
\text { المراجـع }
$$

الجنـايني، عصــماء عــادل 2000. دراسـة تحليليـة

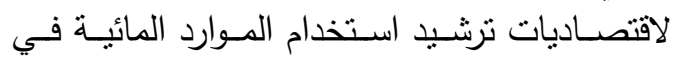

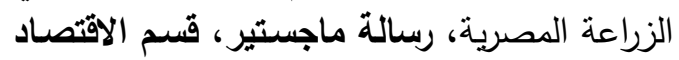
الزراعـي، كليـة الزراعـة ، جامعــة القـاهرة، 112

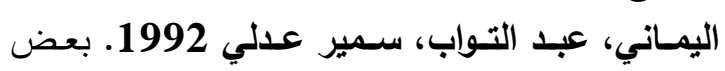
قضايا استخدام الموارد الأرضية والمائية في مصر ، 


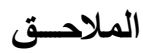

ملحق 1. عرض الموارد المائية المتاحة (مليار متر مكعب) خلال الفترة (2006-2016)

\begin{tabular}{|c|c|c|c|c|c|c|c|}
\hline المرال المائية & تلحية & واللسيول & الصرير مياه & الصرف النزاعي & الجالجوفية & مصاه نهز & السنوات \\
\hline 69.56 & 0.06 & 1.30 & 1.20 & 5.40 & 6.10 & 55.5 & 2006 \\
\hline 69.96 & 0.06 & 1.30 & 1.30 & 5.70 & 6.10 & 55.5 & 2007 \\
\hline 72.36 & 0.06 & 1.30 & 1.30 & 8.00 & 6.20 & 55.5 & 2008 \\
\hline 73.65 & 0.05 & 1.10 & 1.10 & 9.65 & 6.25 & 55.5 & 2009 \\
\hline 73.35 & 0.05 & 1.30 & 1.25 & 8.95 & 6.30 & 55.5 & 2010 \\
\hline 72.69 & 0.05 & 1.30 & 1.30 & 8.24 & 6.30 & 55.5 & 2011 \\
\hline 74.16 & 0.06 & 0.63 & 1.30 & 9.17 & 7.50 & 55.5 & 2012 \\
\hline 75.40 & 0.06 & 0.74 & 1.30 & 10.10 & 7.70 & 55.5 & 2013 \\
\hline 76.00 & 0.10 & 0.90 & 1.30 & 11.50 & 6.70 & 55.5 & 2014 \\
\hline 76.40 & 0.10 & 0.90 & 1.30 & 11.70 & 6.90 & 55.5 & 2015 \\
\hline 76.36 & 0.12 & 1.04 & 1.30 & 11.90 & 6.50 & 55.5 & 2016 \\
\hline 73.63 & 0.07 & 1.07 & 1.27 & 9.12 & 6.60 & 55.5 & المتوسط \\
\hline
\end{tabular}

المصدر : وزارة الموارد المائية والري، الجهاز المركزي للتعبئة العامة والاحصاء، الكتاب الإحصائي السنوي، أعداد متفرقة. 


$$
\text { السبد - عبد المقصود - عرفه }
$$

ملحق 2. استخدامات الموارد المائية والطلب عليها في مختلف القطاعات الاقتصادية خلال الفترة (2006-

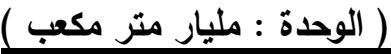

\begin{tabular}{|c|c|c|c|c|c|c|}
\hline إجمالي الطلب & النهلاحة & الصناعة & والاستخدامات & من النيل والتترع بالتبخر & الزراعة & السنوات \\
\hline 68.55 & 0.20 & 1.15 & 6.10 & 2.10 & 59.00 & 2006 \\
\hline 69.25 & 0.20 & 1.15 & 6.50 & 2.10 & 59.30 & 2007 \\
\hline 70.23 & 0.20 & 1.33 & 6.60 & 2.10 & 60.00 & 2008 \\
\hline 73.80 & 0.20 & 1.20 & 9.00 & 2.10 & 61.30 & 2009 \\
\hline 74.05 & 0.20 & 1.20 & 9.35 & 2.00 & 61.30 & 2010 \\
\hline 73.95 & 0.20 & 1.20 & 9.55 & 2.10 & 60.90 & 2011 \\
\hline 74.70 & 0.20 & 1.20 & 9.60 & 2.20 & 61.50 & 2012 \\
\hline 75.70 & 0.20 & 1.20 & 9.70 & 2.50 & 62.10 & 2013 \\
\hline 76.20 & 0.20 & 1.20 & 9.95 & 2.50 & 62.35 & 2014 \\
\hline 76.60 & 0.20 & 1.20 & 10.35 & 2.50 & 62.35 & 2015 \\
\hline 77.33 & 0.20 & 1.20 & 10.65 & 2.50 & 62.78 & 2016 \\
\hline 73.67 & 0.20 & 1.20 & 8.85 & 2.25 & 61.17 & المتوسط \\
\hline
\end{tabular}

المصدر : وزارة الموارد المائية والري - الجهاز المركزي للتعبئة العامة والاحصاء، الكتاب الإحصائي السنوي، أعداد متفرقة. 
ملحق 3. متوسط نصيب الفرد من مياه النيل ومساحة الأراضي الزراعية والتعداد السكاني في مصر خلال الفترة (2016-2006)

\begin{tabular}{|c|c|c|c|c|}
\hline بالمليون نسمةد السكاني & $\begin{array}{c}\text { الأراضي الزراعية } \\
\text { (ألف فدانة) } \\
\end{array}$ & سنوبط نصيب الفرد من مياه النيل & 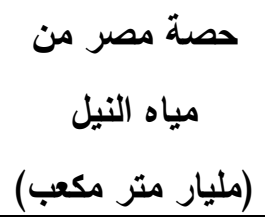 & السنوات \\
\hline 72.01 & 8411 & 770.74 & 55.5 & 2006 \\
\hline 73.64 & 8423 & 753.63 & 55.5 & 2007 \\
\hline 75.19 & 8432 & 738.10 & 55.5 & 2008 \\
\hline 76.93 & 8783 & 721.48 & 55.5 & 2009 \\
\hline 78.68 & 8741 & 705.35 & 55.5 & 2010 \\
\hline 80.53 & 8619 & 689.19 & 55.5 & 2011 \\
\hline 82.55 & 8799 & 672.32 & 55.5 & 2012 \\
\hline 84.63 & 8954 & 655.80 & 55.5 & 2013 \\
\hline 86.81 & 8916 & 639.30 & 55.5 & 2014 \\
\hline 87.96 & 9096 & 630.95 & 55.5 & 2015 \\
\hline 89.80 & 9131 & 618.02 & 55.5 & 2016 \\
\hline 76.03 & 8530 & 738.13 & 55.5 & المتوسط \\
\hline
\end{tabular}

المصدر : وزارة الموارد المائية والري - الجهاز المركزي للتعبئة العامة والاحصاء، الكتاب الإحصائي السنوي، أعداد متفرقة 


\title{
ECONOMETRIC MODEL OF DETERMINANTS OF THE EGYPTIAN WATER RESOURCES
}

\author{
El-Sayed $^{1^{\star}}$ R.E.M., AbdelMaksoud ${ }^{1}$ A.M. and Arafa ${ }^{2}$ Y.E.H. \\ 1- Agric. Economics Dept., Fac. of Agric., Ain Shams Univ., P.O. Box 68, Hadayek Shobra \\ 11241, Cairo, Egypt \\ 2- Agric. Engineering Dept., Fac. of Agric., Ain Shams Univ., P.O. Box 68, Hadayek Shobra \\ 11241, Cairo, Egypt
}

[159]

${ }^{*}$ Corresponding author: ramdan.alsyd2000@gmail.com

Received 26 June, 2018

Accepted 28 August, 2018

\section{ABSTRACT}

The water issue has taken over all Egyptian efforts to try to overcome all obstacles to water supply in the short and long run. Especially in the light of contemporary changes, especially the construction of the Al-Nahda Dam, and therefore the aim of the research to identify the most important determinants of water resources in Egypt, in preparation for inclusion in a proposed mechanism for the promotion of water resources in Egypt.

The research aimed at analyzing the economics of water resources in Egyptian agriculture to identify the most important economic determinants facing the Egyptian water resources sector in general and the water uses in Egyptian agriculture in particular, as well as the most important strengths, weaknesses, opportunities and threats facing the Egyptian water sector.
The results of the statistical assessment of the econometric model of the Egyptian water resource determinants under the influence of water policies show that the supply of available water resources is increasing by about 210 million cubic meters due to the Egyptian government adopting water policies aimed at rationalizing the use of water in all aspects of life, The repercussions of the construction of the Al-Nahda Dam have been in recent times. The most important of these policies is the reduction of cultivated areas of water crops, the development of plant varieties and varieties of strategic crops with lower water consumption rates, the expansion of the application of wastewater treatment technology, the expansion and adoption of modern irrigation techniques, and the lining and clearing of canals, waterways and other policies. Especially in recent times.

Keywords: Economics of water resources, Econometric model, Water policy

$$
\text { تحكيم: ا.د محمد عبد الصادق السنتريسى الد عبد اله عاصم كريم عبد الحميد }
$$

